OPEN ACCESS

Edited by:

Pietro Spataro,

Mercatorum University, Italy

Reviewed by:

Karin Matko,

Technische Universität Chemnitz,

Germany

Srikanth N. Jois,

World Pranic Healing Foundation

India, India

*Correspondence:

Apar Avinash Saoji

aparsaoj@gmail.com

Specialty section:

This article was submitted to

Cognition,

a section of the journal

Frontiers in Psychology

Received: 09 September 2021

Accepted: 22 November 2021

Published: 17 December 2021

Citation:

Swathi PS, Bhat $R$ and Saoji AA

(2021) Effect of Trataka (Yogic Visual

Concentration) on the Performance in

the Corsi-Block Tapping Task: A

Repeated Measures Study.

Front. Psychol. 12:773049.

doi: 10.3389/fpsyg.2021.773049

\section{Effect of Trataka (Yogic Visual Concentration) on the Performance in the Corsi-Block Tapping Task: A Repeated Measures Study}

\author{
P. S. Swathi, Raghavendra Bhat and Apar Avinash Saoji* \\ Division of Yoga and Life Sciences, Swami Vivekananda Yoga Anusandhana Samsthana, Bengaluru, India
}

Background and Objective: Attention and memory are essential aspects of cognitive health. Yogasanas, pranayama, and meditation have shown to improve cognitive functions. There has been no assessment of Trataka (yogic visual concentration) on working or on spatial memory. The present study was planned to assess the immediate effects of Trataka and of eye exercise sessions on the Corsi-block tapping task (CBTT).

Methods: A total of 41 healthy volunteers of both genders with age $23.21 \pm 2.81$ years were recruited. All participants underwent baseline assessment, followed by 2 weeks of training in Trataka (including eye exercise). Each training session lasted for $20 \mathrm{~min} /$ day for 6 days a week. After completion of the training period, a 1-week washout period was given. Each participant then was assessed in two sessions in Trataka and in eye exercise on two separate days, maintaining the same time of the day. Repeated measure analysis of variance with Holm's adjustment was performed to check the difference between the sessions.

Results: Significant within-subjects effects were observed for forward Corsi span andforward total score $(p<0.001)$, and also for backward Corsi span $(p<0.05)$ and backward total score $(p<0.05)$. Post hoc analyses revealed Trataka session to be better than eye exercises and baseline. The eye exercise session did not show any significant changes in the CBTT.

Conclusion: The result suggests that Trataka session improves working memory, spatial memory, and spatial attention.

Keywords: Trataka, yoga, shatkriya, kriya, Corsi-block tapping task, eye exercise, cognition, spatial memory

\section{INTRODUCTION}

Yoga, an ancient Indian tradition, is aimed at all-round personality development (Taimni, 2010). The practices in the discipline of yoga include yama and niyama (moral and ethical conduct), asana (physical postures), pranayama (regulated breathing), dharana, dhyana (meditation), and shuddhikriya (cleansing practices). Scientific research in recent times has explored the positive impact of yoga practices on various domains of physiology and psychology in healthy and therapeutic settings (Field, 2016). One major area of interest in yoga research has been the effects of yoga practices on cognition and performance. Yoga practices appear to prevent neurodegeneration and enhance neuroplasticity by influencing specific brain areas involved with domains of cognition 
such as hippocampus, amygdala, prefrontal cortex, insula, and default mode network (Marciniak et al., 2014; Gothe et al., 2019). A meta-analysis, which included fifteen RCTs and eight acute exposure studies, indicated the beneficial effect of yoga on cognition, attention, processing speed, and memory (Gothe and McAuley, 2015).

Various aspects of cognition, such as spatial and visual memory scores (Joshi and Telles, 2008; Garg et al., 2016; Gupta et al., 2019), verbal memory (Naveen et al., 1997), executive functions, attention, and concentration (Chattha et al., 2008), working memory (Subramanya and Telles, 2009), response inhibition (Rajesh et al., 2014), visual attention (Jarraya et al., 2019), and task-switching (Anusuya et al., 2021), were found to be positively influenced through yoga practices such as yogasanas, pranayama, and meditation techniques. Yoga practice was found to be better than physical exercises in improving cognitive functions in school children (Vhavle et al., 2019).

The classical texts of Hathayoga described the profound impact of the six cleansing techniques on various aspects of one's personality, which are also validated through empirical studies (Muktibodhananda, 1999; Swathi et al., 2020). Trataka (Yogic Visual concentration) is one of the cleansing techniques considered to enhance vision and positively influence cognitive processes. Since the process of Trataka involves focused attention on a candle flame, the practice leads to the mind becoming one-pointed and arouses inner vision (Muktibodhananda, 1999). Earlier studies on Trataka and cognition have demonstrated enhanced performance in Stroop Task (Raghavendra and Singh, 2016; Sherlee and David, 2020), Six Letter Cancelation, Trail Making tasks (Talwadkar et al., 2014), and Critical Flicker Fusion (Mallick and Kulkarni, 2010). Considering the earlier studies on Trataka and cognition, we hypothesize that Trataka may positively influence the domains of cognition, such as spatial and working memory. Corsi-block tapping task (CBTT) is a neuropsychological test that measures visuospatial short-term and working memory. The task can be performed using a computer to collect the data with precision (Kessels et al., 2000; Siddi et al., 2020). Considering the wide use and ease of administration of CBTT, the current study was designed to evaluate the effect of Trataka on the performance in the CBTT.

\section{MATERIALS AND METHODS}

\section{Participants}

A total of 90 volunteers from a University in South India were briefed about the study protocol. Out of which, 60 consented to participate in the study. The inclusion criteria were normal vision (6/6) on Snellen's chart and regular physical and psychological health as assessed by a physician who, otherwise, had no role in the study. We included participants with prior experience of yoga practices other than Trataka. We excluded volunteers who had any known eye disorders, including refractive errors, color blindness, glaucoma, cataract, any ophthalmological surgeries, or presence of cognitive or neurological disorders, respiratory or cardiac, and sensory abnormalities. We also excluded participants who had a history of smoking or alcoholism. Finally, 41 subjects ( 8 male and 33 female) with their mean ( \pm SD) age $23.21 \pm 2.81$ years were recruited to the study. Out of the 41 subjects, 31 were pursuing their undergraduate education, 4 were graduates, and 6 had completed postgraduation. Their experience in yoga ranged between 1 and 7 years (mean $\pm \mathrm{SD}=3.98 \pm 1.44$ ).

\section{Sample Size Calculation}

The sample size was calculated using $G^{*}$ power where alpha was 0.05 and power was 0.8 . The effect size was found to be 0.50 (Gupta et al., 2019). The recommended sample size resulted in being 33 participants for each session. Considering dropouts to be at about $25 \%$ during the training, we decided to have 41 participants for each session.

\section{Ethical Consideration and Trial Registration}

The Institutional Ethics committee approved the study of the university (Ref. No: RES/IEC-SVYASA/182-C/2021). Written informed consent was obtained from individual participants before their recruitment to the study. The study was registered with the Clinical Trial Registry of India (CTRI/2021/03/031872).

\section{Trial Design}

We executed a within-subject repeated measures design. All participants underwent baseline assessment, followed by 2 weeks of training in Trataka (including eye exercise). Each training session lasted for $20 \mathrm{~min} /$ day for 6 days a week. This orientation was administered to avoid individual variations in the practice. After completion of the training period, a 1-week washout period was given. Each participant then was assessed in two sessions in Trataka and in eye exercise on two separate days, maintaining the same time of the day (between $4 \mathrm{pm}$ and $6 \mathrm{pm}$ ). The order of allotment of Trataka and eye exercise sessions was block randomized using a web-based random number generator ${ }^{1}$. Half the participants practiced Trataka on day 1, eye exercise on day 2 and vice versa. The CBTT was recorded following both the trial conditions (Trataka and eye exercise).

\section{Intervention \\ Baseline}

The participants were asked to give their baseline assessment without any intervention. On the day of baseline assessment, the participants were seated comfortably in a cross-legged position for 5 min prior to the commencement of the CBTT performance.

\section{Trataka Session}

Each Trataka session consisted of 20 min practice. Throughout the practice, the participants were seated comfortably on the floor in a cross-legged position. The practice consists of 2 distinct stages. Each Trataka session involved a preparatory stage of eye exercises for $10 \mathrm{~min}$. These were performed with eyes open in a well-lit, soundproofed recording room in the laboratory. During this stage, the participants were asked to move the eyeballs in horizontal, vertical, diagonal, and circular directions. The second

\footnotetext{
${ }^{1}$ www.randomizer.org
} 
stage is the practice of gazing at the candle flame in a dark room, where the candle was placed at the participant's eye level at a distance of $2 \mathrm{~m}$. The participants were asked to fix their gaze on the candle flame for about 2 to 3 min without blinking their eyes. Then they were asked to visualize the candle flame between the eyebrows with closed eyes. This process was repeated for three rounds. Later, subjects were asked to defocus, and practice concluded in silence with a prayer. This stage lasted for a total duration of $10 \mathrm{~min}$. A pre-recorded audio was used to maintain uniformity of the practice among participants.

\section{Eye Exercise Session}

The eye exercise session included eyeball movements in the horizontal, vertical, diagonal, and circular directions for $10 \mathrm{~min}$ followed by $10 \mathrm{~min}$ of quiet sitting with closed eyes. The eyes open part was performed in well-lit room, while the eyes closed part was performed by switching off the lights to maintain similarity of interventions.

\section{Assessments Corsi-Block Tapping Task}

Corsi-block tapping task is a popular neuro-psychological task used to assess working and spatial memory. Nine blue squares appear on the screen. For each trial, the squares "light up" as yellow one by one in a varying sequence. After the presentation, the participants had to click each of the boxes in a similar order in which they have to "lit up" the first part of the task, i.e., forward tapping. In the second part of the task, they maintained the reverse order, i.e., backward tapping (Kessels et al., 2008). The task begins with a two-box sequence to a maximum of nine. The test gets terminated when the participant cannot remember the sequence for two consecutive trials at any one level. Hence, the test assesses the following four variables: (i) forward Corsi span, (ii) forward total score, (iii) backward Corsi span, and (iv) the backward total score. Figure 1 illustrates the forward and backward CBTT.

\section{Presentation of Corsi-Block Tapping Task}

We assessed the participants at baseline, following Trataka and eye exercise sessions. The participants were asked to avoid caffeine consumption on all the assessment days as it may alter their cognitive abilities. The CBTT (Kessels et al., 2000, 2008) was presented using the INQUISIT software package 4.0 (Millisecond Software, LLC, Seattle, WA, United States) on a Dell desktop computer with a 21.5 color monitor. Uniform configuration was maintained for the computers on which the CBTT was presented to maintain the uniform processing speed. All participants received a practice session prior to the actual assessment session to familiarize themselves with the CBTT. The experiment was conducted individually in a room under standard fluorescent lighting in the research laboratory.

\section{Data Analysis}

The data were tabulated and data analyses were performed using JASP statistical package version $0.14 .1^{2}$. The data were tested

${ }^{2}$ https://jasp-stats.org for normality and repeated measures (RM) ANOVA for withinsubjects effects. Post hoc corrections were done using Holm's method for checking the differences between sessions.

\section{RESULTS}

All 41 participants (eight male) completed all three sessions. RM ANOVA demonstrated significant within-subjects effect in Forward Corsi Span $F(2,80)=8.757, p<0.001$; Forward total scores $F(2,80)=11.377, p<0.001$; Backward Corsi Span $F(2,80)=3.629, p=0.031$; Backward total scores $F(2,80)=3.950$, $p=0.023$. The within-subjects effects obtained through RM ANOVA are presented in Table 1.

Pairwise comparisons between the sessions performed through RM ANOVA with Holm's corrections demonstrated significantly higher scores following Trataka sessions when compared with baseline for Forward Corsi Span, $t=-4.11$, $p<0.001$; Forward Total Score, $t=-4.76, p<0.001$; and Backward Total Score, $t=-2.74, p<0.05$. Scores following the Trataka session were significantly higher than following Eye exercises for Forward Corsi Span, $t=2.74, p<0.05$; Forward Total Score, $t=2.65, p<0.05$. The scores increased from baseline, following Eye exercise only for Forward Total Scores, $t=-2.10, p<0.05$. The effect sizes and $t$-values for between sessions using RM ANOVA with Holm's correction along with the group mean and SD are reported in Table 2.

\section{DISCUSSION}

The current study was designed to elicit if the practice of Trataka affects the working and the spatial memory through the performances in the CBTT. All the four measures, viz., forward and backward Corsi spans, and total scores, demonstrated significance within the subject's effect. The Corsi span and total scores were higher following Trataka while comparing with baseline and Eye exercises. Scores following eye exercises and baseline sessions were insignificant except in Forward total score. The forward span and total score of CBTT measure materialspecific slave systems. The backward test measures primarily tax central executive resources (Monaco et al., 2013). Thus, improvements in both forward and backward span and total scores indicated a positive effect of Trataka on working, spatial memory, and executive functions while comparing with baseline and eye exercises.

Earlier studies on Trataka and cognition have shown improvements in the domains of selective attention, cognitive flexibility, and response inhibition through the Stroop task (Raghavendra and Singh, 2016; Sherlee and David, 2020). Another study shown improvement in the performance of critical flicker fusion after the immediate practice of Trataka in 35 volunteers (Mallick and Kulkarni, 2010). After the practice of Trataka for 26 days, the performances of the digit span test, the six-letter cancelation test, and the trail making test significantly improved in thirty elderly subjects compared to the waitlist control group (Talwadkar et al., 2014). Thus, improvements 


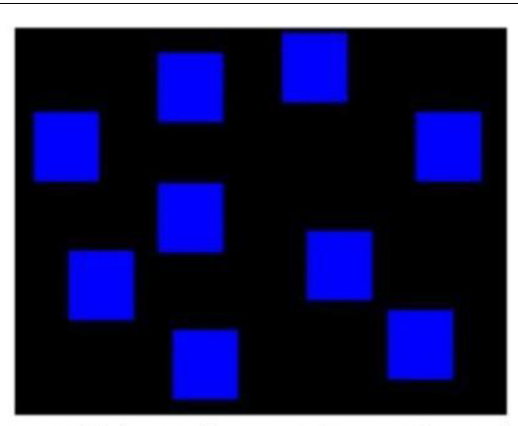

Nine blue squares which are shown at the starting point of CBTT
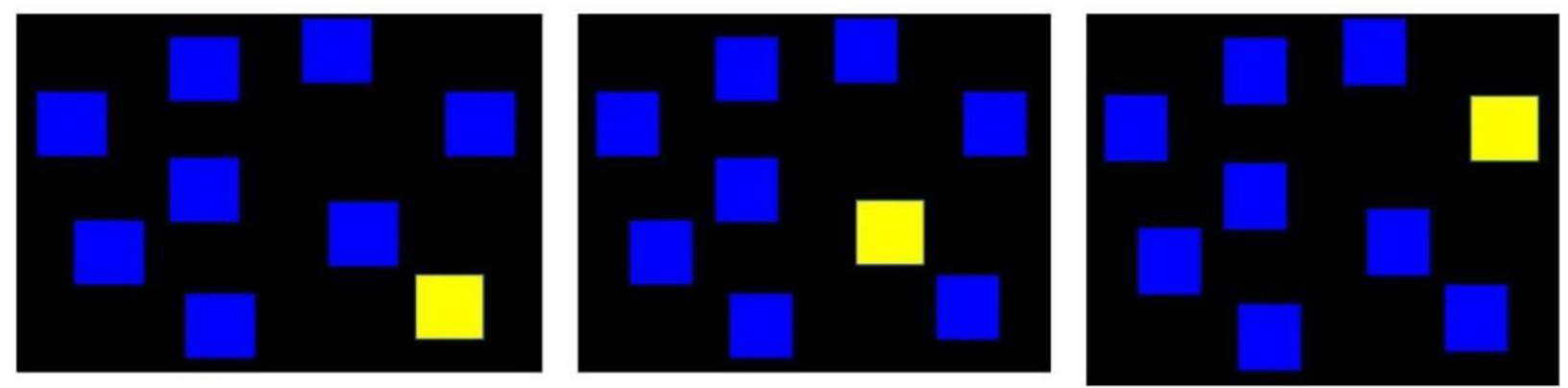

Sequence of the square light up as yellow

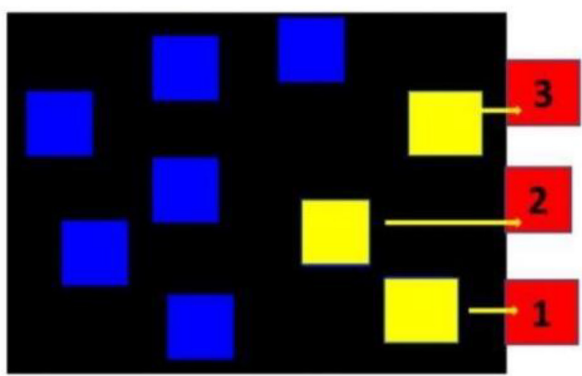

Forward tapping - click on the same order

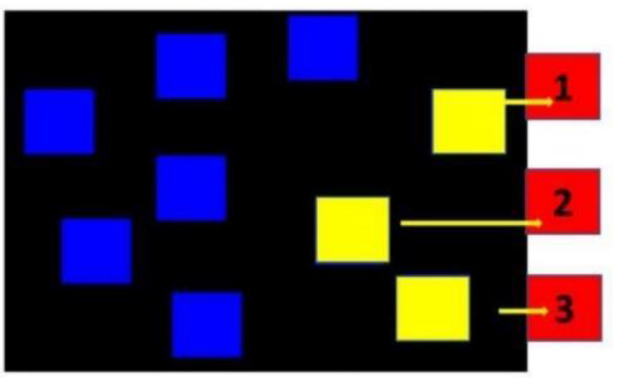

Backward tapping - click on the reverse order

FIGURE 1 | Corsi-block tapping task (CBTT): Forward and backward.

TABLE 1 | Results of repeated measures analysis of variance for within-subjects effects.

\begin{tabular}{lcccc}
\hline Variable & $\boldsymbol{F}$ & $\mathbf{d f}$ & $\boldsymbol{P}$ & Partial $\eta^{2}$ \\
\hline Forward Corsi span & 8.757 & 2,80 & $<0.001$ & 0.180 \\
Forward total score & 11.377 & 2,80 & $<0.001$ & 0.221 \\
Backward Corsi span & 3.629 & 2,80 & $=0.031$ & 0.083 \\
Backward total score & 3.950 & 2,80 & $=0.023$ & 0.090 \\
\hline
\end{tabular}

noted in our study in the cognitive abilities following Trataka are similar to the earlier studies.

Trataka practice is indicated to positively influence cognition from both classical texts of yoga (Muktibodhananda, 1999) and empirical studies (Swathi et al., 2020). Although classified as a cleansing procedure, the practice of Trataka is similar to focused meditation techniques. Earlier studies on meditation for a total duration of 8 weeks shown decreased negative mood and enhanced attention, working memory, and decreased state anxiety on the trier social stress test (TSST) in population naive to meditation practice (Basso et al., 2019). Another focused attention meditation showed significant improvement in working memory in the reading span test and in activation of bilateral dorsolateral prefrontal cortex (DLPFC) during the intervention in the experimental group (Yamaya et al., 2021). Similarly, other Yoga interventions showed improved cognitive communicative abilities (Namratha et al., 2017).

The improvement in the performance of CBTT may have been mediated through relaxation, attained through the 


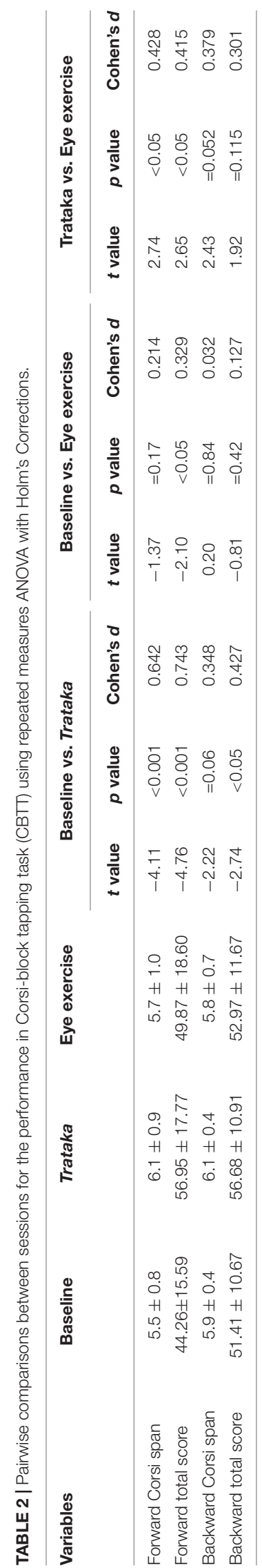

practice of Trataka (Raghavendra and Ramamurthy, 2014). The possible mechanisms for improving working and spatial memory following the Trataka session could be the process of Trataka itself, involving focused attention. This focused attention is also elaborated in the Yoga Sutras (aphorisms) of Patanjali (Taimni, 2010). A recent study has also demonstrated enhanced mindfulness, attention, and reduced mind-wandering with the practice of Trataka (Swathi et al., 2021). Thus, improved working memory found in our study could be due to a reduction in mind-wandering and enhanced focusing. The prefrontal cortex is associated with memory, attention, executive functions, and various other complex cognitive functions (Miller, 2000). Thus, the results following the Trataka session could be due to activation of the prefrontal cortex. However, further studies with neuroimaging techniques may be required to confirm this mechanism of action.

Another possible mechanism could be a surge in melatonin release due to practice in the dim light. It is known that bright light tunes the suprachiasmatic nucleus (SCN) that regulates the circadian rhythm. Exposure to bright light impedes the melatonin synthesis, whereas the dim light initiates the surge in melatonin release (Zisapel, 2018). Melatonin has been found to positively influence learning and memory (Zakaria et al., 2016). Thus, further studies on Trataka may assess the serum melatonin levels as one of the variables.

Our study indicated a beneficial role of Trataka in enhancing the CBTT performance in healthy volunteers. CBTT performance is commonly altered in neurodegenerative disorders such as early-stage Parkinson's (Stoffers et al., 2003) and Alzheimer's disease (Guariglia, 2007). Thus, future studies may be planned in a clinical population, where the CBTT performance is compromised.

Using a repeated measures design for immediate effect is one of the strengths of the study. We also used a computerbased program to execute CBTT, which enabled robust results (Brunetti et al., 2014). The limitation of the study includes not incorporating a neuro-imaging technique, which has limited our ability to predict the exact mechanism of action. Thus, future studies on Trataka and cognitive performance should include neuroimaging techniques. Another major limitation of the study is control condition which had eye exercise for $10 \mathrm{~min}$ followed by $10 \mathrm{~min}$ of quiet sitting in which they were told not to meditate. However, we are not sure if during quiet sitting, if they focused on breathing or let their mind wandered freely. We could not get an equal number of male and female participants and were also unable to study the impact of long-term practice of tataka. Lastly, the effect of Trataka in a population with mild cognitive decline could be studied in future.

\section{CONCLUSION}

The results of this study indicated a positive impact of the Trataka session on the CBTT, indicating enhanced working memory, spatial memory, and spatial attention among the subjects compared to the baseline and eye exercise sessions. 
Thus, Trataka could be used to improve memory and attention in young adults.

\section{DATA AVAILABILITY STATEMENT}

The raw data supporting the conclusions of this article will be made available by the authors, without undue reservation.

\section{ETHICS STATEMENT}

The studies involving human participants were reviewed and approved by Swami Vivekananda Yoga Anusandhana Samsthana. The patients/participants provided their written informed consent to participate in this study.

\section{REFERENCES}

Anusuya, U. S., Mohanty, S., and Saoji, A. A. (2021). Effect of mind sound resonance technique (MSRT-A yoga-based relaxation technique) on psychological variables and cognition in school children: a randomized controlled trial. Complement. Ther. Med. 56:102606. doi: 10.1016/j.ctim.2020. 102606

Basso, J. C., McHale, A., Ende, V., Oberlin, D. J., and Suzuki, W. A. (2019). Brief, daily meditation enhances attention, memory, mood, and emotional regulation in non-experienced meditators. Behav. Brain Res. 356, 208-220. doi: 10.1016/j. bbr.2018.08.023

Brunetti, R., Del Gatto, C., and Delogu, F. (2014). eCorsi: implementation and testing of the Corsi block-tapping task for digital tablets. Front. Psychol. 5:939. doi: 10.3389/fpsyg.2014.00939

Chattha, R., Nagarathna, R., Padmalatha, V., and Nagendra, H. R. (2008). Effect of yoga on cognitive functions in climacteric syndrome: a randomised control study. BJOG 115, 991-1000. doi: 10.1111/j.1471-0528.2008.01749.x

Field, T. (2016). Yoga research review. Complement. Ther. Clin. Pract. 24, 145-161. doi: 10.1016/j.ctcp.2016.06.005

Garg, R., Malhotra, V., Tripathi, Y., and Agarawal, R. (2016). Effect of left, right and alternate nostril breathing on verbal and spatial memory. J. Clin. Diagn. Res. 10, CC01-CC03. doi: 10.7860/JCDR/2016/12361.7197

Gothe, N. P., and McAuley, E. (2015). Yoga and cognition: a meta-analysis of chronic and acute effects. Psychosom. Med. 77, 784-797. doi: 10.1097/PSY. 0000000000000218

Gothe, N. P., Khan, I., Hayes, J., Erlenbach, E., and Damoiseaux, J. S. (2019). Yoga effects on brain health: a systematic review of the current literature. Brain Plasticity 5, 105-122. doi: 10.3233/BPL- 190084

Guariglia, C. C. (2007). Spatial working memory in Alzheimer's disease: a study using the Corsi block-tapping test. Dement. Neuropsychol. 1, 392-395. doi: 10.1590/S1980-57642008DN10400011

Gupta, R., Agnihotri, S., Telles, S., and Balkrishna, A. (2019). Performance in a Corsi block-tapping task following high-frequency yoga breathing or breath awareness. Int. J. Yoga 12:247. doi: 10.4103/ijoy.ijoy_55_18

Jarraya, S., Wagner, M., Jarraya, M., and Engel, F. A. (2019). 12 weeks of Kindergarten-based yoga practice increases visual attention, visual-motor precision and decreases behavior of inattention and hyperactivity in 5-year-old children. Front. Psychol. 10:796. doi: 10.3389/fpsyg.2019.00796

Joshi, M., and Telles, S. (2008). Immediate effects of right and left nostril breathing on verbal and spatial scores. Indian J. Physiol. Pharmacol. 52, 197-200.

Kessels, R. P. C., Van Den Berg, E., Ruis, C., and Brands, A. M. A. (2008). The backward span of the corsi block-tapping task and its association with the WAIS-III digit span. Assessment 15, 426-434. doi: 10.1177/107319110831 5611

Kessels, R. P. C., Van Zandvoort, M. J. E., Postma, A., Kappelle, L. J., and De Haan, E. H. F. (2000). The corsi block-tapping task: standardization and normative data. Appl. Neuropsychol. 7, 252-258. doi: 10.1207/S15324826AN0704_8

\section{AUTHOR CONTRIBUTIONS}

PS was involved in conceptualization, execution, data collection, and writing and editing of the manuscript. RB was involved in conceptualization, data analysis and interpretation, and writing and editing of the manuscript. AS was involved in conceptualization, data collection, analysis and interpretation, and writing and editing of the manuscript. All authors contributed to the article and approved the submitted version.

\section{FUNDING}

The authors gratefully acknowledge Swami Vivekananda Yoga Anusandhana Samsthana (Deemed to be University), Bengaluru, for the funding.

Mallick, T., and Kulkarni, R. (2010). The effect of Trataka, a yogic visual concentration practice, on critical flicker fusion. J. Altern. Complement. Med. 16, 1265-1267. doi: 10.1089/acm.2010.0012

Marciniak, R., Sheardova, K., Čermáková, P., Hudeček, D., Šumec, R., and Hort, J. (2014). Effect of meditation on cognitive functions in context of aging and neurodegenerative diseases. Front. Behav. Neurosci. 8:17. doi: 10.3389/fnbeh. 2014.00017

Miller, E. K. (2000). The prefrontal cortex and cognitive control. Nat. Rev. Neurosci. 1, 59-65. doi: 10.1038/35036228

Monaco, M., Costa, A., Caltagirone, C., and Carlesimo, G. A. (2013). Forward and backward span for verbal and visuo-spatial data: standardization and normative data from an Italian adult population. Neurol. Sci. 34, 749-754. doi: 10.1007/ s10072-012-1130-x

Muktibodhananda, S. (1999). Hatha Yoga Pradipika. Munger: Yoga Publications trust.

Namratha, H. G., George, V. M., Bajaj, G., Mridula, J., and Bhat, J. S. (2017). Effect of yoga and working memory training on cognitive communicative abilities among middle aged adults. Complement. Ther. Clin. Pract. 28, 92-100. doi: 10.1016/J.CTCP.2017.05.007

Naveen, K. V., Nagarathna, R., Nagendra, H. R., and Telles, S. (1997). Yoga breathing through a particular nostril increases spatial memory scores without lateralized effects. Psychol. Rep. 81, 555-561. doi: 10.2466/pr0.1997.81.2.555

Raghavendra, B. R., and Ramamurthy, V. (2014). Changes in heart rate variability following yogic visual concentration (Trataka). Heart India 2:15.

Raghavendra, B. R., and Singh, P. (2016). Immediate effect of yogic visual concentration on cognitive performance. J. Tradit. Complement. Med. 6, 34-36. doi: 10.1016/j.jtcme.2014.11.030

Rajesh, S., Ilavarasu, J., and Srinivasan, T. (2014). Effect of bhramari pranayama on response inhibition: evidence from the stop signal task. Int. J. Yoga 7:138. doi: 10.4103/0973-6131.133896

Sherlee, J. I., and David, A. (2020). Effectiveness of yogic visual concentration (Trataka) on cognitive performance and anxiety among adolescents. J. Complement. Integr. Med 17. doi: 10.1515/jcim-2019-0055 [Epub ahead of print].

Siddi, S., Preti, A., Lara, E., Brébion, G., Vila, R., Iglesias, M., et al. (2020). Comparison of the touch-screen and traditional versions of the Corsi blocktapping test in patients with psychosis and healthy controls. BMC Psychiatry 20:329. doi: 10.1186/s12888-020-02716-8

Stoffers, D., Berendse, H. W., Deijen, J. B., and Wolters, E. C. (2003). Deficits on Corsi's block-tapping task in early stage Parkinson's disease. Parkinsonism Relat. Disord. 10, 107-111. doi: 10.1016/s1353-8020(03)00106-8

Subramanya, P., and Telles, S. (2009). Effect of two yoga-based relaxation techniques on memory scores and state anxiety. Biopsychosoc. Med. 3:8. doi: 10.1186/1751-0759-3-8

Swathi, P. S., Raghavendra, B. R., and Saoji, A. A. (2020). Health and therapeutic benefits of shatkarma: a narrative review of scientific studies. J. Ayurveda Integr. Med. 12, 206-212. doi: 10.1016/j.jaim.2020.11.008 
Swathi, P. S., Saoji, A. A., and Raghavendra, B. (2021). Role of Trataka in ameliorating visual strain and promoting psychological well-being during prolonged use of digital displays: randomized controlled trial. Work A J. Prev. Assess. Rehabil. (in press).

Taimni, I. K. (2010). The Science of Yoga: The Yoga-sutras of Patanjali in Sanskrit with Transliteration in Roman, Translation in English and Commentary. Quest Books Available online at: https://books.google.com/ books?id=q9kenQEACAAJ\&pgis=1 (accessed April 4, 2016).

Talwadkar, S., Jagannathan, A., and Raghuram, N. (2014). Effect of trataka on cognitive functions in the elderly. Int. J. Yoga 7, 96-103. doi: 10.4103/09736131.133872

Vhavle, S., Rao, R., and Manjunath, N. (2019). Comparison of yoga versus physical exercise on executive function, attention, and working memory in adolescent schoolchildren: a randomized controlled trial. Int. J. Yoga 12:172. doi: 10.4103/ ijoy.ijoy_61_18

Yamaya, N., Tsuchiya, K., Takizawa, I., Shimoda, K., Kitazawa, K., and Tozato, F. (2021). Effect of one-session focused attention meditation on the working memory capacity of meditation novices: a functional near-infrared spectroscopy study. Brain Behav. 11:e2288. doi: 10.1002/brb3.2288

Zakaria, R., Ahmad, A. H., and Othman, Z. (2016). The potential role of melatonin on memory function: lessons from rodent studies. Folia Biol. 62, 181-187.
Zisapel, N. (2018). New perspectives on the role of melatonin in human sleep, circadian rhythms and their regulation. Br. J. Pharmacol. 175, 3190-3199. doi: 10.1111/BPH.14116

Conflict of Interest: The authors declare that the research was conducted in the absence of any commercial or financial relationships that could be construed as a potential conflict of interest.

Publisher's Note: All claims expressed in this article are solely those of the authors and do not necessarily represent those of their affiliated organizations, or those of the publisher, the editors and the reviewers. Any product that may be evaluated in this article, or claim that may be made by its manufacturer, is not guaranteed or endorsed by the publisher.

Copyright $\odot 2021$ Swathi, Bhat and Saoji. This is an open-access article distributed under the terms of the Creative Commons Attribution License (CC BY). The use, distribution or reproduction in other forums is permitted, provided the original author(s) and the copyright owner(s) are credited and that the original publication in this journal is cited, in accordance with accepted academic practice. No use, distribution or reproduction is permitted which does not comply with these terms. 